\title{
STUDY ON LAMB WAVE PROPAGATION CHARACTERISTICS ALONG THE GRAIN OF THIN WOOD SHEET
}

\author{
Minghua Wang, Ming Li, Tingting Deng, Saiyin Fang, Xiaosong Li, Fei Lai \\ Southwest Forestry University \\ China \\ (Received April 2020)
}

\begin{abstract}
Through the time-frequency analysis of the propagation waveform of the acoustic emission (AE) signal propagating in the thin sheet of Pinus sylvestris var. mongolica, the propagation characteristics of the stress wave when propagating as a lamb wave was studied. An AE source was generated on the surface of the specimen, the discrete wavelet transform method was used to achieve AE signal de-noising and reconstruct the waveform of the AE signal. On this basis, the time difference positioning method was used to calculate the propagation velocity of lamb waves, and compared with the propagation characteristics of lamb waves in the metal specimen. The results show that the high-frequency mode of lamb waves attenuated sharply as they propagate in the thin wood sheet, indicating that the microstructure of wood has a significant low-pass characteristic for lamb waves. The average attenuation rates of lamb waves in metal and thin wood sheet were $87.1 \%$ and $75.7 \%$, and the velocity was $4447.0 \mathrm{~m} \cdot \mathrm{s}^{-1}$ and $1186.3 \mathrm{~m} \cdot \mathrm{s}^{-1}$, respectively. This shows that AE signals can travel longer distances in the thin wood sheet, but the propagation velocity is significantly reduced.
\end{abstract}

KEYWORDS: Wood, acoustic emission, lamb wave, propagation characteristics, discrete wavelet transform.

\section{INTRODUCTION}

In thin plate members, stress waves propagate mainly in the form of lamb waves. Lamb wave is essentially a special form of stress waves formed by multiple couplings of longitudinal and transverse waves in a plate and have a strong ability to propagate, so they are mainly used for health monitoring of thin plate structures (Ai et al. 2012, Jiang et al. 2016). Using lamb waves to detect defects in plate-shaped structural parts has been one of the research hotspots in the field of active detection technology (Zhang et al. 2008, Wang et al. 2018). 
Hou (2009) and Chen et al. (2011) used lamb waves to effectively identify the surface defects of aluminum plates. Chen et al. (2019) found that lamb wave images based on time inversion can intuitively reflect the damage location and defect shape of carbon fiber composite laminates. In order to achieve effective detection of near-field defects in aluminum thin plates, Zhang et al. (2019) proposed a method combining Green's function inversion theory with lamb wave array imaging. Gao et al. (2018) proposed a smooth Capon algorithm to estimate the exact path length of wave packets for the problem of low resolution of defect detection caused by lamb wave dispersion. Soleimanpour et al. (2016) studied the scattering behavior of lamb waves at cracks in aluminum plates and found that the scattering characteristics of A0 mode lamb waves are closely related to the size and direction of the notches. Han et al. (2015) proposed a time-frequency beam detection method based on lamb waves to achieve effective localization of structural defects in aluminum plates. For structural defect location, Yu et al. (2012) proposed a hyperbolic location algorithm. Lee et al. $(2007 \mathrm{a}, \mathrm{b})$ used the amplitude attenuation and transmission time difference of lamb waves to characterize the degree of damage to the material structure and achieve defect localization. These studies have effectively promoted the development of AE technology based on stress wave analysis in the field of metal detection. In addition, the AE technology has also been applied to the study of damage detection of wood materials, such as the mechanical properties of wood under external load (Niemz et al. 2009, Krauss et al. 2011), classification and performance prediction of thermally modified wood (Nasir et al. 2019a, 2019b), identification defects of wood materials (Sun et al. 2008, 2013), etc. However, up to now, the research on lamb wave focuses more on isotropic materials such as aluminum plates, while the research on typical anisotropic materials such as wood, which is widely used, is relatively rare.

Wooden board is a common complex biomass material with the characteristics of both composite materials and porous materials, and its physical properties in all directions are significantly different (Zhao et al. 2011). Using the AE technique to study the propagation characteristics of lamb waves in specific directions on boards without damage defects and to investigate the effectiveness of the technique in this application will help to extend the application of the $\mathrm{AE}$ technique to active damage monitoring. In addition, this can lay a theoretical foundation for the future use of guided waves for defect detection in wooden boards. At present, the most common parameters used to characterize the structural changes of a plate using stress waves are time-frequency characteristics, $\mathrm{AE}$ wave velocity, $\mathrm{AE}$ energy, and so on. Therefore, this paper takes Pinus sylvestris sawn timber as the research object and explores the time-frequency characteristics and propagation law of lamb waves as they propagate along the grain direction of the wood. A 2-channel AE signal acquisition system based on NI high-speed acquisition equipment was used to obtain the original $\mathrm{AE}$ signal on the surface of the specimen, the discrete wavelet transform method and normalization process were used to reconstruct the $\mathrm{AE}$ signal waveform that was "submerged" in noise. Furthermore, according to the time-frequency characteristics of the reconstructed signal, the effect of the microstructure of the wood along the grain direction on the characteristics of the lamb wave signal was studied. Then used signal correlation analysis and time difference location methods to calculated the propagation velocity of lamb along the grain direction of the wood, and compared it with carbon steel of the same size to further studied the influence of wood microstructure on lamb wave propagation velocity and energy attenuation rate. 


\section{MATERIAL AND METHODS}

\section{Materials}

In this paper, two sets of specimens were made using Pinus sylvestris sawn timber and Q235 carbon steel sheet respectively and tested independently. The size of both specimens was $580 \times 55 \times 4 \mathrm{~mm}$. For carbon steel, the modified size refers to length, width and thickness respectively, and for wood it is axial, tangential and radial.

Based on NI USB-6336 high-speed acquisition card and Lab VIEW software to build a 2-channel AE signal acquisition platform, the maximum sampling frequency of each channel is $2 \mathrm{MHz}$. The existing research ( $\mathrm{Li}$ et al. 2018, Shen et al. 2015) shows that the maximum frequency of $\mathrm{AE}$ signal of wood was approximately $200 \mathrm{kHz}$, in order to effectively collect the original AE signals with wide band distribution, the SR-150N single resonance AE sensor with a bandwidth of $22 \mathrm{kHz} \sim 220 \mathrm{kHz}$ was used. At the same time, a preamplifier with a gain of $40 \mathrm{~dB}$ was configured, and its response frequency is $10 \mathrm{kHz}$ to $2 \mathrm{MHz}$. According to the Shannon sampling theorem, in order to restore the analog signal without distortion, the sampling frequency $f_{s}$ and the maximum frequency $f_{\text {max }}$ of the signal must satisfy $f_{s} \geq 2 . f_{\max }$, therefore, the sampling frequency of each channel was set to $500 \mathrm{kHz}$ during the experiment.

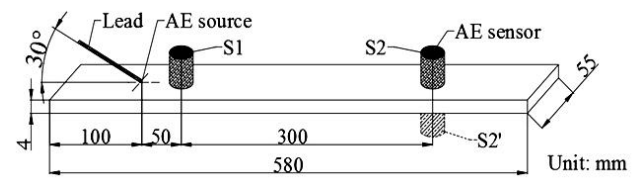

Fig. 1: AE sensor layout diagram.

\section{Methods}

An artificial AE source was generated by a pencil lead break at a position of $100 \mathrm{~mm}$ on the left end of the specimen. Sensors S1 and S2 were spaced $300 \mathrm{~mm}$ apart on the surface of the specimen. Among them, sensor S1 was placed $50 \mathrm{~mm}$ from the AE source $(150 \mathrm{~mm}$ from the left end of the specimen), the sensor S2 adopted two arrangements of the same side (S2 of Fig. 1) and the opposite side (S2' of Fig. 1) with the sensor S1 to collected the AE signal on the surface of the specimen. In order to minimize the influence of the two sides of the specimen on the $\mathrm{AE}$ signal propagation, the $\mathrm{AE}$ source and the sensor were placed on the central axis in the width direction according to the above positions, as shown in Fig. 1. The experiment was divided into two groups, the first group was the Pinus sylvestris var. mongolica specimen and the second was the carbon steel metal plate specimen. The experimental operation refers to the American ASTM-E976 standard to ensure the consistency of the AE source during the experiment, that was, the $2 \mathrm{H}$ resin lead with a diameter of $0.5 \mathrm{~mm}$ was placed at an angle of $30^{\circ}$ with the surface of the specimen, and it was broken at a distance of $2.5 \mathrm{~mm}$ from the contact point, to obtain original AE signals on the surfaces of both specimens. At the same time, in order to fully reduce the influence of the air medium on the experiment, high-temperature vacuum insulation silicone grease was filled between the specimen and the sensor. In addition, the sensor was fixed on the surface of the specimen with a rubber band.

The original AE signal contains a lot of noise signals. The discrete wavelet transform method has an excellent de-noising effect and time-frequency localization analysis ability, which can realize signal de-noising and reconstruct $\mathrm{AE}$ waveforms at the same time ( $\mathrm{Li}$ et al. 2019, 2020). Therefore, this paper used the Daubechies wavelet as the discrete wavelet transform 
basis function for the 5-level wavelet decomposition. In the original signal acquisition process, the sampling frequency $f_{s}$ of each channel was $500 \mathrm{kHz}$, so the analysis frequency $f_{b}$ of each channel was $f_{h}=f_{s} / 2=250 \mathrm{kHz}$; after the 5-level wavelet decomposition, the frequency bands of the high-frequency detail signal of each level were $(125 \mathrm{kHz}, 250 \mathrm{kHz}),(62.5 \mathrm{kHz}, 125 \mathrm{kHz})$, $(31.25 \mathrm{kHz}, 62.5 \mathrm{kHz}),(15.625 \mathrm{kHz}, 31.25 \mathrm{kHz}),(7.8125 \mathrm{kHz}, 31.25 \mathrm{kHz})$, covering the entire measurement range of $\mathrm{AE}$ sensors.

The two-point time difference location and signal correlation analysis methods were used to calculate the propagation velocity of the $\mathrm{AE}$ signal. In the experiment, the two sensors were placed in a straight line at a fixed distance $\Delta s=300 \mathrm{~mm}$. After measuring the time difference $\Delta t$ that the reconstructed AE signal arrived at the two sensors using the cross-correlation function, the propagation velocity $\mathrm{v}$ of the $\mathrm{AE}$ signal on the surface of the specimen can be calculated according to the formula $v=\Delta s / \Delta t$. For two random signals $x(t)$ and $y(t)$, the cross-correlation function $R_{x y}(\tau)$ between them was defined as shown in Eq. 1:

$$
R_{x y}(\tau)=\lim _{T \rightarrow \infty} \frac{1}{T} \int_{0}^{T} x(t) y(t+\tau) d t
$$

The cross-correlation function can effectively reduce the influence of random factors in the AE signal. If $\tau=\tau 0$, the absolute value of the cross-correlation function is maximized, which means that after the random signal $y(t)$ was translated by $\tau_{0}$ units on the time axis, the similarity with the random signal $x(t)$ reached the highest level. At this time, $\tau_{0}$ was the time difference $\Delta t$ when the AE signal reached the sensors S1 and S2.

\section{RESULTS AND DISCUSSION}

\section{Characteristics of AE signals on the same side of the specimen}

The AE source generated by artificially breaking the lead core was characterized by a significant signal mutation and a short duration, so the signal components that reflect the $\mathrm{AE}$ characteristics should appear in high-frequency detail part (Ju et al. 2018, 2019). According to the time-frequency characteristics of the signals of each level after decomposition, the detail signal of the second and third levels were selected to reconstruct the signal waveform. The waveform and spectrum of the reconstructed AE signal on the same side of the specimen as shown in Fig. 2.

In terms of time-domain waveform, in the Pinus sylvestris var. mongolica specimen (Fig. 2a), the signal amplitude of sensors S1 and S2 attenuates to a certain extent, which shows that the natural porous microstructure along the grain direction of wood has a certain obstruction on the $\mathrm{AE}$ beam. So the farther away from the AE source, the smaller the energy per unit area, and the lower the AE signal amplitude detected by the sensor. When the AE signal propagates in the thin plate specimen, the transverse and longitudinal waves were repeatedly reflected and overlapped to form a lamb wave, so the waveform appeared as a "wave packet", and the wave packet characteristic becomes more prominent as the propagation distance increases. Therefore, at the end of signal attenuation, the signal waveform detected by sensor S1 shows a small "wave packet" shape, and sensor S2 shows a large "wave packet" shape. In the carbon steel sheet specimen (Fig. 2c) the propagation medium of stress waves has a higher density, the internal friction of particles per unit area is much stronger than that of wood, and the energy consumption is larger, so AE signal not only has amplitude attenuation like that of wood but also shows the phenomenon that amplitude attenuation was more rapid and the degree of attenuation was larger. 

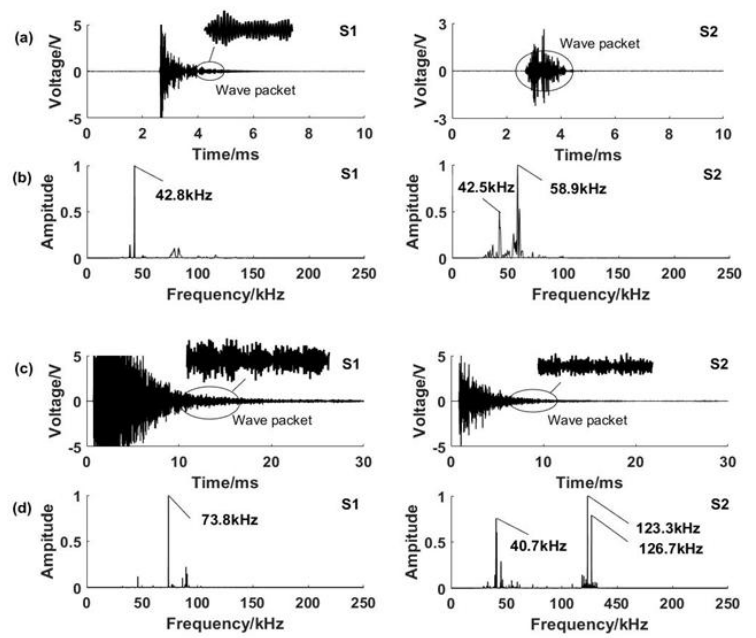

Fig. 2: Reconstructed signal waveform and spectrum on the same side of the specimen: (a) reconstructed signal waveform on the same side of Pinus sylvestris var. mongolica, (b) reconstructed signal spectrum on the same side of Pinus sylvestris var. mongolica, (c) reconstructed signal waveform on the same side of carbon steel, (d) reconstructed signal spectrum on the same side of carbon steel.

Comparing the time-domain waveform of the two materials (Fig. 2a,c), it was found that the "wave packets" of metal and wood were extremely different. The sensor S1 and S2 only show a small "wave packet" in the late stage of propagation attenuation when AE signal propagated in the metal plate, while the wave packet of the sensor S2 was more conspicuous than the sensor S1 when AE signal propagated in thin wood sheet. The reason is that the microstructure of wood is much more complex than that of metal, the interior of wood is full of cavities, the reflection of $\mathrm{AE}$ signal in it is relatively disordered, and there is also scattering caused by medium. Therefore, the formed lamb wave pattern is relatively complicated, and the signal waveform at different positions is greatly different. However, the metal's microstructure is relatively uniform, and the transverse and longitudinal wave have comparatively regular reflections, so there is no significant difference in the AE signal waveform between the two sensors except the amplitude attenuation caused by energy exchange.

Lamb wave is a special stress wave with multi-modal effects, and many different modes will be generated during the forward propagation. In the Pinus sylvestris var. mongolica specimen, the signal of the sensor S1 was distributed among a frequency band centered on $42.8 \mathrm{kHz}$, the sensor S2 had two distribution centers of $42.5 \mathrm{kH}$ and $58.9 \mathrm{kHz}$, and the peak frequency was $58.9 \mathrm{kHz}$. Moreover, the spectral component of S2 was significantly more complicated than that of sensor S1. It means that the frequency of the main mode of the AE signal in the sensor S1 was $42.8 \mathrm{kHz}$, while in the sensor $\mathrm{S} 2$, the lamb wave undergoes a mode transition, and a variety of lamb waves of different modes were generated, and the frequencies of the main modes were $42.5 \mathrm{kH}$ and $58.9 \mathrm{kHz}$. In the metal plate specimen, the central frequency of the main lamb wave mode in sensor $\mathrm{S} 1$ was $73.8 \mathrm{kHz}$, and there were other modes of lamb wave at a frequency band of $73.8 \mathrm{kHz}$ as the distribution center. Similarly, the lamb wave's model changed during the propagation process, so the sensor S2 detected three lamb waves with different frequencies of $40.7 \mathrm{kHz}, 123.3 \mathrm{kHz}$, and $126.7 \mathrm{kHz}$, respectively. 
Comparing Fig. 2b and Fig. 2d, it can be seen that the degree of modal change of the lamb wave in the thin wood sheet was smaller than that of the metal, and only the middle and low-frequency lamb waves were generated during the propagation process. Pinus sylvestris var. mongolica is an anisotropic coniferous wood, and on the micro-scale, it is a porous material composed of hexagonal tracheids, which are mainly thin and long axial tracheids with striate holes in the longitudinal direction (Wittel et al. 2004). Such a microstructure makes it demonstrates a certain "selection" effect on the propagation band of lamb waves and is sensitive to high-frequency modal lamb waves. However, the microscopic composition of metal is relatively uniform, which has a small obstruction to the frequency band of lamb waves (in Fig. 2, the frequency band distribution of AE signal in the metal plate is wider than that in thin wood sheet). This "selection" effect determines that there were differences in the lamb wave's modal changes when the lamb wave propagates in wood and metal. In addition, the spectral composition is also related to the density of the material (Yang et al. 2007). The density of the metal plate is larger and the structure is regular. When the AE signal propagates in metal plate, the reflection of the stress wave is relatively regular in any direction. In addition to the anisotropy of the wood, which causes stress waves to be reflected more turbulently, there are also $\mathrm{AE}$ attenuation caused by changes in the medium. The difference in the form of stress wave propagation and the density of the medium also causes the difference in the spectral components.

\section{Characteristics of AE signals on the opposite side of the specimen.}

In order to further study the characteristics of the lamb wave, the discrete wavelet transform method was used to reconstruct the AE signal when the sensor was placed on the opposite side. The waveform and spectrum of the reconstructed AE signal were shown in Fig. 3.
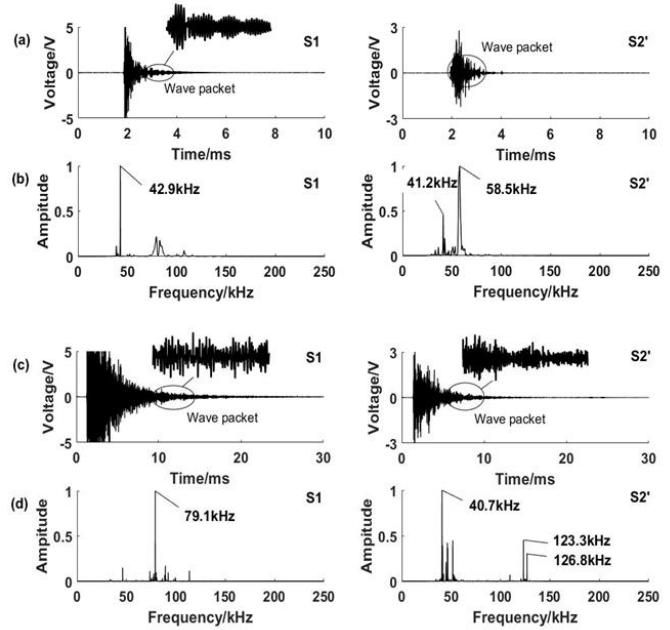

Fig. 3: Reconstructed signal waveform and spectrum on the opposite side of the specimen: (a) reconstructed signal waveform on the opposite side of Pinus sylvestris var. mongolica, (b) reconstructed signal spectrum on the opposite side of Pinus sylvestris var. mongolica, (c) reconstructed signal waveform on the opposite side of carbon steel, (d) reconstructed signal spectrum on the opposite side of carbon steel. 
When the sensor was placed on the opposite side, due to the influence of the microstructure difference of the material itself, the AE signal has a larger energy loss in the metal plate, and the attenuation rates and the degree of the signal were more intense than those of the thin wood sheet. Moreover, the time-domain waveform of the signals collected by the two sensors in the metal plate has a certain similarity, but the time-domain waveform of the signals collected by the two sensors in the wood was tremendous differences. In the thin wood sheet (Fig. 3b), the frequency distribution center of the main mode of the lamb wave detected by the sensor S1 was $42.9 \mathrm{kHz}$. During the forward propagation process, the mode of the lamb wave has changed, resulting in the phenomenon that the lamb wave was distributed over the two frequency bands centered at $42.9 \mathrm{kHz}$ and $58.59 \mathrm{kHz}$ on the sensor S2'.As a result of the change of the Lamb mode in the metal plate (Fig. 3d), the center frequency of the main mode of the sensor S1 was $79.1 \mathrm{kHz}$, and the frequency centers of the three main modes of the sensor S2' were $40.7 \mathrm{kHz}$, $123.3 \mathrm{kHz}$, and $126.8 \mathrm{kHz}$. In addition, influenced by the physical characteristics of materials and the differences in reflected wave patterns, the frequency of the main lamb wave modes in thin wood sheet appeared in the low and medium frequency bands, and the mode change degree was smaller than that of metal.

Comparing with Fig. 2 and Fig. 3, it is not difficult to find a phenomenon: In the two samples, whether the sensor S2' was placed on the same side or on the opposite side of the specimen, there was no remarkable difference in the main mode of the lamb wave in the corresponding sensor. This is mainly because lamb wave is a two-dimensional body wave in nature, with small attenuation and long propagation distance compared with the three-dimensional body wave (Fan et al. 2011). Therefore, when the lamb waves propagate to the same location, their modes will not change obviously, no matter the upper side or lower side of the same position, the spectrum difference of the AE signal is small.

\section{The propagation velocity of AE signals on the surface of the two specimens.}

When calculating the propagation velocity and energy attenuation rate of $\mathrm{AE}$ signal, in order to minimize the effect of random errors on the experiment, 10 independent pencil lead broken tests were performed on the surface of the metal plate and the Pinus sylvestris var. mongolica specimen. Tab. 1 and Tab. 2 were the test results when the sensor was placed on the same side and the opposite side respectively.

Tab. 1: AE signal propagation velocity from $S 1$ to $S 2$.

\begin{tabular}{|c|c|c|c|c|c|c|}
\hline \multirow{2}{*}{$\begin{array}{c}\text { Experiment } \\
\text { number }\end{array}$} & \multicolumn{3}{|c|}{ Pinus sylvestris var.mongolica } & \multicolumn{3}{c|}{ Carbon steel } \\
\cline { 2 - 7 } & $\Delta t(\mathrm{us})$ & $v\left({\left.\mathrm{~m} \cdot \mathrm{s}^{-1}\right)}^{-1}\right.$ & $\eta(\%)$ & $\Delta t(\mathrm{us})$ & $v\left(\mathrm{~m} \cdot \mathrm{s}^{-1}\right)$ & $\eta(\%)$ \\
\hline 1 & 276.0 & 1087.0 & 76.9 & 64.0 & 4687.5 & 84.8 \\
\hline 2 & 292.0 & 1027.4 & 76.4 & 68.0 & 4411.8 & 87.8 \\
\hline 3 & 224.0 & 1339.3 & 76.3 & 64.0 & 4687.5 & 83.6 \\
\hline 4 & 210.0 & 1428.6 & 72.5 & 62.0 & 4838.7 & 89.8 \\
\hline 5 & 224.0 & 1339.3 & 74.4 & 64.0 & 4687.5 & 79.2 \\
\hline 6 & 276.0 & 1087.0 & 75.0 & 62.0 & 4838.7 & 84.6 \\
\hline 7 & 224.0 & 1339.3 & 77.5 & 64.0 & 4687.5 & 88.5 \\
\hline 8 & 274.0 & 1094.9 & 75.1 & 68.0 & 4411.8 & 84.0 \\
\hline 9 & 250.0 & 1200.0 & 78.4 & 66.0 & 4545.5 & 83.9 \\
\hline 10 & 276.0 & 1087.0 & 79.7 & 64.0 & 4687.5 & 85.4 \\
\hline Average value & 252.6 & 1203.0 & 76.2 & 64.6 & 4648.4 & 85.2 \\
\hline
\end{tabular}


Tab. 2: AE signal propagation velocity from $S 1$ to $S 2$ '.

\begin{tabular}{|c|c|c|c|c|c|c|}
\hline \multirow{2}{*}{$\begin{array}{c}\text { Experiment } \\
\text { number }\end{array}$} & \multicolumn{2}{|c|}{ Pinus sylvestris var. mongolica } & \multicolumn{3}{c|}{ Carbon steel } \\
\cline { 2 - 7 } & $\Delta t(\mathrm{us})$ & $v\left(\mathrm{~m} \cdot \mathrm{s}^{-1}\right)$ & $\eta(\%)$ & $\Delta t(\mathrm{us})$ & $v\left(\mathrm{~m} \cdot \mathrm{s}^{-1}\right)$ & $\eta(\%)$ \\
\hline 1 & 290.0 & 1034.5 & 73.9 & 72.0 & 4166.7 & 85.0 \\
\hline 2 & 234.0 & 1282.1 & 70.0 & 66.0 & 4545.5 & 90.2 \\
\hline 3 & 288.0 & 1041.7 & 80.8 & 64.0 & 4687.5 & 87.5 \\
\hline 4 & 232.0 & 1293.1 & 72.8 & 70.0 & 4285.7 & 84.2 \\
\hline 5 & 234.0 & 1282.1 & 73.2 & 64.0 & 4687.5 & 90.7 \\
\hline 6 & 234.0 & 1282.1 & 75.0 & 68.0 & 4411.8 & 82.8 \\
\hline 7 & 288.0 & 1041.7 & 79.9 & 72.0 & 4166.7 & 84.6 \\
\hline 8 & 234.0 & 1282.1 & 78.1 & 64.0 & 4687.5 & 86.2 \\
\hline 9 & 288.0 & 1041.7 & 74.5 & 66.0 & 4545.5 & 89.8 \\
\hline 10 & 234.0 & 1282.1 & 78.4 & 70.0 & 4285.7 & 89.6 \\
\hline Average value & 255.6 & 1186.3 & 75.7 & 67.6 & 4447.0 & 87.1 \\
\hline
\end{tabular}

In Tabs. 1 and 2: $\Delta t$ - the time difference of propagation of the AE signal; $v$ - the propagation velocity; $\eta$ - the energy attenuation rate.

According to Tab. 1, when the sensors S1 and S2 were placed on the same side of the specimens, the average propagation velocity and the average energy attenuation rate of the $\mathrm{AE}$ signals along the texture direction of Pinus sylvestris var. mongolica are $1203.0 \mathrm{~m} \cdot \mathrm{s}^{-1}$ and $76.2 \%$ respectively, while in the metal sheet, the average propagation velocity of the AE signal is $4648.4 \mathrm{~m} \cdot \mathrm{s}^{-1}$, and the average energy attenuation rate is $85.2 \%$. The propagation velocity and energy attenuation of AE signals in the metal specimen was greater than those in the wood specimen. This is because the density of the metal plate is large and the number of particles per unit area is larger, so there is a large energy exchange between the lamb wave and the medium when it propagates in it, and only a large energy loss can be used to ensure the propagation velocity of the stress wave. In addition, the differences in the microstructure of the specimens also result in that the propagation mode of $\mathrm{AE}$ wave in the thin wood sheet was more intricate than that in the metal specimen, including lamb waves and a small part of scattering waves caused by complex internal boundaries. However, the $\mathrm{AE}$ wave in the metal plate is transmitted in the form of a purer lamb wave than the thin wood sheet. The difference in the $\mathrm{AE}$ waveform is also one of the reasons for the difference in the propagation velocity and energy attenuation of the AE signal in the two specimens.

When the sensor was placed on the opposite side of the specimens (Tab. 2), it was also due to the differences in the materials and the different $\mathrm{AE}$ waveform, the average propagation velocity and the average energy attenuation rate of the AE signal in the thin wood sheet are $1186.3 \mathrm{~m} \cdot \mathrm{s}^{-1}$ and $75.7 \%$ respectively, while in the metal sheet, the average propagation velocity of the AE signal is $4447.0 \mathrm{~m} \cdot \mathrm{s}^{-1}$, and the average energy attenuation rate is $87.1 \%$. Comparing with Tabs. 1 and 2, it can be seen that in the same specimen, no matter how the sensor was placed, there was no prominent difference in the propagation velocity and energy attenuation of signal. This also shows that the $\mathrm{AE}$ wave propagates forward in the form of lamb waves on the plate, so the same position of the same specimen, the signal's components, propagation velocity, and energy attenuation of the upper and lower sides of the specimens will not be greatly different. 


\section{CONCLUSIONS}

This paper refers to the ASTM-E976 standard to generated an AE source, and studies the propagation characteristics of $\mathrm{AE}$ signals in two typical materials, and explores the differences in spectral characteristics and propagation velocity of lamb waves when it propagated in wood and metal. Preliminary explorations have shown that the AE technique based on lead core fracture is capable of being used to study the propagation characteristics of Lamb waves in wooden boards. Affected by the physical properties of the material, the propagation form of the AE signal is extremely complicated when it propagates in the thin wood sheet, which includes lamb waves and scattered waves caused by the discontinuous interface in the interior of the wood. So the time-domain waveform at different locations were significantly different. In the metal plate, the $\mathrm{AE}$ signal propagates in the form of lamb waves, so in addition to amplitude attenuation, the time-domain waveform at different locations have certain similarities.

The lamb waves will produce a variety of signal components in dissimilar modes during the propagation process, which results in the complexity of the signal's spectrum components and appear multiple distribution centers with an increase of the propagation distance. The interior structure of wood has a strong resistance to the high-frequency modes of lamb waves, and the reflection of stress waves in them is relatively chaotic. However, the metal plate has a uniform structure and has a small obstruction to each frequency band of the AE signal, and the boundary reflection is relatively regular. As a result, the lamb wave has a relatively small modal change in the thin wood sheet compared with the metal and only produces lamb waves at the low and middle frequency bands.

The crystals inside the metal plate are compactly arranged, and the vibration between particles in unit area is stronger than that of wood. Therefore, when Lamb propagates in it, it gets a higher propagation velocity in the form of higher energy consumption. However, the structure of the wood is relatively loose, and there are cavities and micro-discontinuous interfaces inside, which will cause some scattering of the stress wave. As a result, the lamb wave uses a lower energy consumption and a smaller propagation velocity to ensure that the signal can achieve a longer distance spread. Lamb wave is essentially a two-dimensional body wave and has a strong ability to propagate, so there is no predominant difference in signal components, propagation velocity, and energy attenuation between the upper and lower sides of the same material at the same location of the same specimen.

In this paper, the $\mathrm{AE}$ characteristics of the lamb wave propagating along the direction of the wood grain were initially explored. However, as a typical anisotropic material, wood has significant differences in mechanical properties in all directions. Therefore, using AE technology to further study the acoustic characteristics of wood in other directions has clear practical significance for the rational use of wood. Based on this study, the effect of wood anisotropy on AE signal propagation characteristics can be further explored in the future.

\section{ACKNOWLEDGMENTS}

The authors are grateful to the National Natural Science Foundation of China (NO: 31760182, NO: 31100424) and Department of Education of Yunnan Provincial (NO: 2017ZZX215, NO: 2020Y0376, NO: 2021J0156, NO: 2021J0158, NO: 2021Y224) for funding this research. 


\section{REFERENCES}

1. Ai, C.A., Wang, X.Y., Liu,Y., 2012: Ultrasonic detection of lamb wave stimulation and modal analysis of thin steel plate structure. Noise and Vibration Control 32(1): 137-140.

2. Cao, X., Zeng, L., Lin, J., Hua, J.D., 2018: Improving the resolution for lamb wave testing via a smoothed Capon algorithm. Measurement science and Technology 29(4): 1-26.

3. Chen, J., Li, Z.H., Lin, L., Hou, Y.X., Ma, Q.J., 2011: Experiment investigations of lamb waves in an aluminum plate. Applied Acoustics 30(2): 98-104.

4. Chen, Y., Zhang, B.Y., Wu, X., Chen, G., Shi, W.Z., 2019: Air-coupled lamb waves imaging for impact damage of CFRP plates based on the time reversal damage index. Failure Analysis and Prevention 14(01): 5-11.

5. Fan, S.B., Yan, X.Q. Fu, M.F., Xie, Z.L., Yu, L., 2011: The research on locating method of acoustic emission source based on the dispersive characteristics of lamb wave in sheet. Chinese Journal of Solid Mechanics (S1): 283-287.

6. Han, J.H., Kim, Y.J., 2015: Time-frequency beam forming for nondestructive evaluations of plate using ultrasonic Lamb wave. Mechanical Systems \& Signal Processing 54-55: 336-356.

7. Hou, Y.X., Chen, J., Lin, L., 2009: Mode identification and application for nondestructive evaluation of thin aluminum sheet by lamb waves. Nondestructive testing 31(7): 517-519.

8. Jiang, Y,D., Zhang, L.W., Yang, G.B., 2016: Load localization method for a metallic plate based on lamb wave. Nondestructive Testing 38(4): 26-30.

9. Ju, S., Li, X.C., Luo, T.F., Li, M., 2018: Characteristics of acoustic emission signals on the surface of Masson pine glulam with wavelet analysis method. Journal of Northeast Forestry University 46(08): 86-92.

10. Ju, S., Li, X.C., Luo, T.F., Li ,M., 2019: Anisotropic propagation of acoustic emission signal on surface of Pinus massoniana Lamb. glulam. China Forestry Science and Technology 4(2): 54-59.

11. Krauss, A., Kudela, J., 2011: Ulrasonic wave propagation and Young's modulus of elasticity along the grain of Scots pine wood (Pinus sylvestris L.) varying with distance from the pith. Wood Research 56(4): 479-488.

12. Lee, B.C., Staszewski, W.J., 2007a: Lamb wave propagation modelling for damage detection (I): two- dimensional analysis. Smart Materials and Structures 16(2): 249-259.

13. Lee, B.C., Staszewski, W.J., 2007b: Lamb wave propagation modelling for damage detection (II): Damage monitoring strategy. Smart Materials and Structures 16(2): 260-274.

14. Li, X.C., Ju, S., Luo, T.F., Li, M., 2019: Influence of adhesive layer at Masson pine glulam on acoustic emission signal propagation characteristics. Journal of Northwest Forestry University 34(3): 185-190.

15. Li, X.C., Ju, S., Luo, T.F., Li, M., 2020: Effect of moisture content on propagation characteristics of acoustic emission signal of Pinus massoniana Lamb. European Journal of Wood and Wood Products 78: 185-191.

16. Li, Y., Luo, T.F., Yu, S.S., Shen, L.J., Li, M., 2017: Signal propagation characteristics of acoustic emission and douglas fir glulam beams. Journal of Northwest Forestry University 32(5): 197-201.

17. Nasir, V., Nourian, S., Avramidis, S., Cool, J., 2019a: Stress wave evaluation for predicting the properties of thermally modified wood using neuro-fuzzy and neural network modeling. Holzforschung 73(9): 827-838. 
18. Nasir, V., Nourian, S., Avramidis, S., Cool, J., 2019b: Stress wave evaluation by accelerometer and acoustic emission sensor for thermally modified wood classification using three types of neural networks. European Journal of Wood and Wood Products 77(1): 45-55.

19. Niemz, P., Brunner A.J., Walter O., 2009: Investigation of the mechanism of failure behaviour of wood based materials using acoustic emission analysis and image processing. Wood Research 54(2): 49-62.

20. Shen, K.N., Zhao, H.L., Ding, X.C., Li, M., 2015: Wavelet analysis of acoustic emission signal in wood damage and fracture process. Journal of Henan University of Science \& Technology (Natural Science) 36(3): 33-37.

21. Soleimanpour, R., Ng, C.T., 2016: Scattering of the fundamental anti-symmetric lamb wave at through-thickness notches in isotropic plates. Journal of Civil Structural Health Monitoring 6(3): 447-459.

22. Sun, J.P., Wang, F.H., Zhu, X.D., 2008: Application of wavelet-neural network in defect location non-destructive testing of MDF. Chinese Journal of Scientific Instrument 29(5): 954-958.

23. Sun, J.P., Hu, Y.C., Wang, F.H., 2013: Study on quantitative nondestructive test of wood defects based on intelligent technology. Chinese Journal of Scientific Instrument 34(9): 1955-1960.

24. Wang, G.P., Shi, Z., Wu, M.L., Wei, M., 2018: Experimental study on damage identification of thin plate structure based on lamb wave. Internal Combustion Engine \& Parts 255(03): 56-58.

25. Wittel, F.K., Dill-Langer, G., Kroplin, B.H., 2004: Modeling of damage evolution in soft-wood perpendicular to grain by means of a discrete element approach. Computational Materials Science 32(3): 594-603.

26. Yang, H.M., Wang, L.H., 2007: Nondestructive testing of wood hole defect by ultrasonic spectrum analysis. Journal of Northeast Forestry University 35(8): 30-32.

27. Yu, L., Cheng, L., Su, Z., 2012: Correlative sensor array and its applications to identification of damage in plate-like structures. Structural Control \& Health Monitoring. 19(8): 650-671.

28. Zhang, H., Zhang, H,Y., Xu, M.Y., Fan, G.P., Zhu, F.W., Chai, X.D., 2019: Lamb waves topological imaging combining with Green's function retrieval theory to detect near filed defects in isotropic plates. Chinese Physics B 28(07): 290-296.

29. Zhang, H.P., Zhu, C.H., 2008: Study of propagational characteristics of lamb wave. Metrology \& Measurement Technology 28(5): 5-9.

30. Zhao, R.J., Chen, X.B., Sun, J., Wang, X.Q., Fei, B.H., 2011: Study on the longitudinal tensile strength of the tracheids of soft wood. Journal of Anhui Agricultural University 38(4): 491-495.

\author{
Minghua Wang, Ming Li*, Tingting Deng, Xiaosong Li \\ Southwest Forestry University \\ School of Machinery and Transportation Engineering \\ KunMing 650224 \\ CHINA \\ *Corresponding author: swfu_lm@swfu.edu.cn
}


